\title{
0-000 FROM REFLECTION DATA TO TRANSMISSION CODA
}

JAN THORBECKE, KEES WAPENAAR and DEYAN DRAGANOV Delft University of Technology, Department of Applied Earth Sciences, Mijnbouwstraat 120, 2628 RX Delft

\section{Summary}

Using the reciprocity theorem of the correlation type an implicit relation between seismic reflection and transmission data has previously been derived. In this paper this relation is used to calculate the transmission coda from reflection data measured at the surface. The transmission response is represented by a generalized propagator consisting of a primary propagator and a coda operator. Using this representation it is possible to solve the implicit relation for the coda operator, using an eigenvalue decomposition on the correlation of the reflection response. The calculated coda response may be used in seismic reflection imaging to obtain an image in which the internal multiple scattering effects are suppressed. A simple example for the estimation of the transmission coda illustrates the discussed procedure.

\section{Introduction}

The one-way reciprocity relation of the correlation type in the frequency domain, introduced by Wapenaar and Grimbergen (1996):

$$
\int_{\partial \mathcal{D}_{0}}\left\{\left(P_{A}^{+}\right)^{*} P_{B}^{+}-\left(P_{A}^{-}\right)^{*} P_{B}^{-}\right\} d^{2} \mathbf{x}_{H}=\int_{\partial \mathcal{D}_{m}}\left\{\left(P_{A}^{+}\right)^{*} P_{B}^{+}-\left(P_{A}^{-}\right)^{*} P_{B}^{-}\right\} d^{2} \mathbf{x}_{H}
$$

is the starting point to derive a relation between reflection and transmission data. The medium parameters in both states $A$ and $B$ are assumed to be identical, lossless and 3-D inhomogeneous and the domain $\mathcal{D}$ is source free. $\partial \mathcal{D}_{0}$ and $\partial \mathcal{D}_{m}$ are two horizontal boundaries at $x_{3,0}$ and $x_{3, m}$, respectively, enclosing the domain $\mathcal{D}$ (see figure (1)). $\mathbf{x}_{H}$ denotes the horizontal coordinates $\left(x_{1}, x_{2}\right)$ and ${ }^{*}$ denotes complex conjugation. Both states, and the related flux-normalized up- and down-going fields $P^{-}$and $P^{+}$respectively, are shown in the table of figure (1). Substituting the two states in the table of figure (1) into equation (1) results in:

$$
\delta\left(\mathbf{x}_{H, A}-\mathbf{x}_{H, B}\right)-\int_{\partial \mathcal{D}_{0}} R_{0}^{*}\left(\mathbf{x}, \mathbf{x}_{A}\right) R_{0}\left(\mathbf{x}, \mathbf{x}_{B}\right) d^{2} \mathbf{x}_{H}=\int_{\partial \mathcal{D}_{m}} T_{0}^{*}\left(\mathbf{x}, \mathbf{x}_{A}\right) T_{0}\left(\mathbf{x}, \mathbf{x}_{B}\right) d^{2} \mathbf{x}_{H} .
$$

Here $R_{0}\left(\mathbf{x}, \mathbf{x}_{A}\right)$ is the reflection response of the inhomogeneous medium in $\mathcal{D}$, including all internal multiples, for a source at $\mathbf{x}_{A}=\left(\mathbf{x}_{H, A}, x_{3,0}\right)$ and a receiver at $\mathbf{x} . T_{0}\left(\mathbf{x}, \mathbf{x}_{A}\right)$ is the transmission response of the inhomogeneous medium in $\mathcal{D}$. The subscript ${ }_{0}$ denotes that no free surface multiples are included. There is no unique way to solve the full transmission response from equation (2). However, by using a suitable representation of the transmission operator there is a way to solve the transmission coda.

\section{Transmission coda calculated from eigenvalues of correlated reflection data}

Equation (2) is rewritten in matrix notation:

$$
\mathbf{T}^{H} \mathbf{T}=\mathbf{I}-\mathbf{R}^{H} \mathbf{R},
$$

where superscript ${ }^{H}$ denotes complex-conjugate transpose. A column of matrix $\mathbf{R}$ contains the discretized version of $R_{0}\left(\mathbf{x}, \mathbf{x}_{A}\right)$ for a fixed source position at $\mathbf{x}_{A}$ and a range of receiver positions $\mathbf{x}$ at $x_{3,0}$ (Berkhout (1982)). I is an identity matrix. For a fixed spread geometry $\mathbf{R}$ is symmetric (but not Hermitian) due to the 


\section{State A}

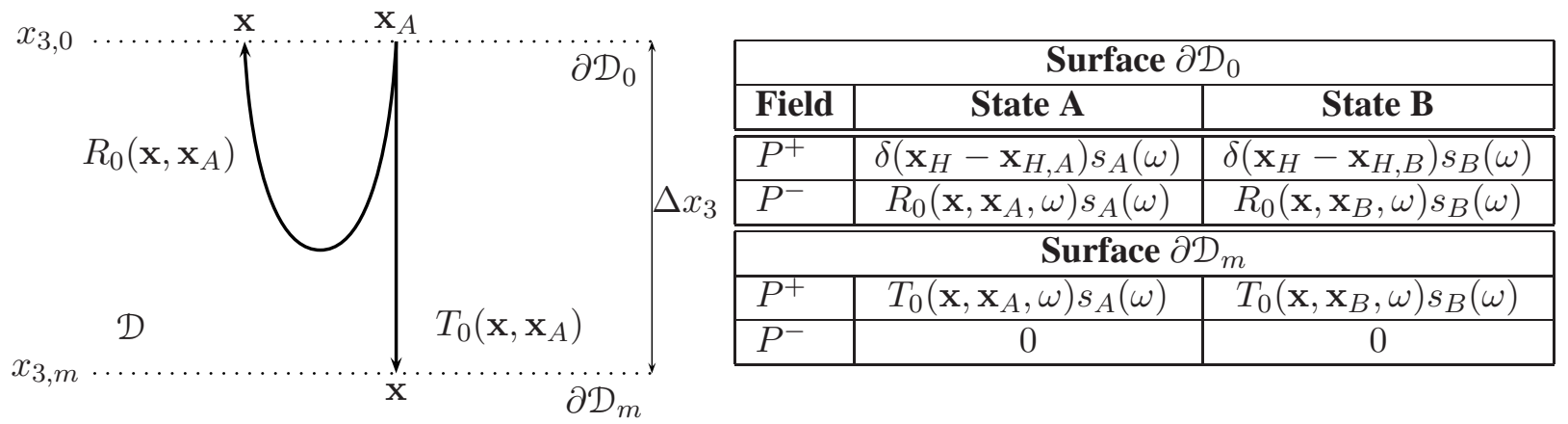

Figure 1: At $\mathbf{x}_{A}$ (state A) or $\mathbf{x}_{B}$ (state B; not shown) just above $\partial \mathcal{D}_{0}$ there is a source for downgoing waves. The half-spaces above $\partial \mathcal{D}_{0}$ and below $\partial \mathcal{D}_{m}$ are homogeneous. The half-space below $\partial \mathcal{D}_{m}$ is source free. The wave fields of both states are shown in the table.

reciprocity relation between source and receiver positions. The transmission response is written as:

$$
\mathbf{T}=\mathbf{W}_{p} \mathbf{C}
$$

where $\mathbf{W}_{p}$ is the primary propagator for downgoing waves between depth levels $x_{3,0}$ and $x_{3, m}$ and where C accounts for the coda due to multiple scattering caused by the inhomogeneities between the two depth levels. Substituting equation (4) into equation (3) and making use of the fact that for the primary propagator we can write $\mathbf{W}_{p}^{H} \mathbf{W}_{p}=\mathbf{I}$, gives

$$
\mathbf{C}^{H} \mathbf{C}=\mathbf{I}-\mathbf{R}^{H} \mathbf{R}
$$

This equation states that the auto-correlation of the transmission coda matrix can be obtained from the autocorrelation of the reflection matrix. To resolve $\mathbf{C}$ from $\mathbf{C}^{H} \mathbf{C}$ the assumption is made that it is possible to decompose $\mathbf{C}$ in its eigenvalues;

$$
\mathbf{C}=\mathbf{L} \boldsymbol{\Lambda}_{c} \mathbf{L}^{H}
$$

with

$$
\boldsymbol{\Lambda}_{c}=\exp \left\{\mathbf{A} \Delta x_{3}\right\}=\left(\begin{array}{cccc}
e^{-\mathcal{A}_{1} \Delta x_{3}} & 0 & \ldots & 0 \\
0 & e^{-\mathcal{A}_{2} \Delta x_{3}} & \ldots & 0 \\
\ldots \ldots & \ldots \ldots \ldots & \ldots & \ldots \\
0 & 0 & \ldots & e^{-\mathcal{A}_{N} \Delta x_{3}}
\end{array}\right)
$$

and $\mathcal{A}_{1}, \mathcal{A}_{2}, \ldots, \mathcal{A}_{N}$ being the temporal Fourier transforms of causal filters. Using the assumption of equation (6) the correlation of the coda matrix of equation (5) can be written as:

$$
\begin{aligned}
& \mathbf{C}^{H} \mathbf{C}=\mathbf{L} \boldsymbol{\Lambda}_{c}^{H} \boldsymbol{\Lambda}_{c} \mathbf{L}^{H} \\
& \boldsymbol{\Lambda}_{c}^{H} \boldsymbol{\Lambda}_{c}=\exp \left\{-2 \mathcal{R}\{\mathbf{A}\} \Delta x_{3}\right\}
\end{aligned}
$$

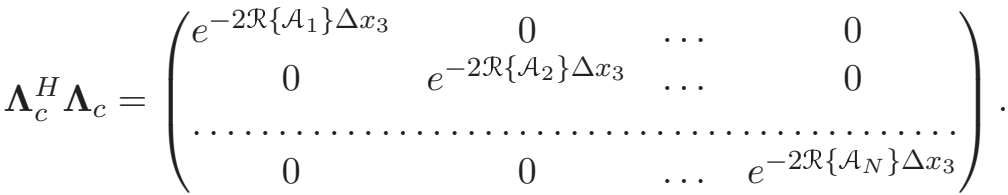

Note that for plane waves in 1D media $\mathbf{C}$ is a circulant matrix, which eigenvalues can be found by using the Fourier transform: $\mathbf{C}=\mathbf{F}^{H} \boldsymbol{\Lambda}_{c} \mathbf{F}$. Furthermore, O'Doherty and Anstey (1971) showed that for 1D media the coda operator $\mathbf{C}$ is related to the exponent of the power spectrum of the reflection coefficient. The elements $\mathcal{A}_{l}$ of $\mathbf{A}$ are the Fourier transforms of causal filters in the time domain. This statement is true for 1D media, since in that case $\mathbf{A}$ is the spatial Fourier transform of $\mathbf{C}$, so $A_{l}=\tilde{\mathcal{A}}\left(k_{x, l}, \omega\right)$. Note that before the temporal Fourier transforms yields the causal filters, the diagonal elements must be scaled from wavenumber $k_{x}$, 


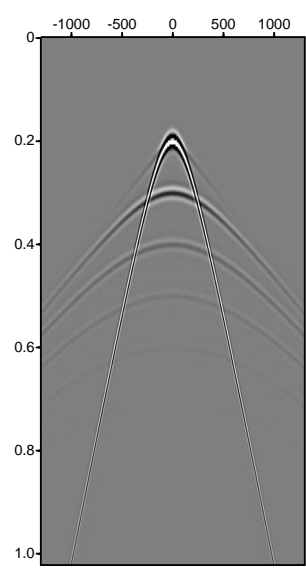

$\mathbf{R}$

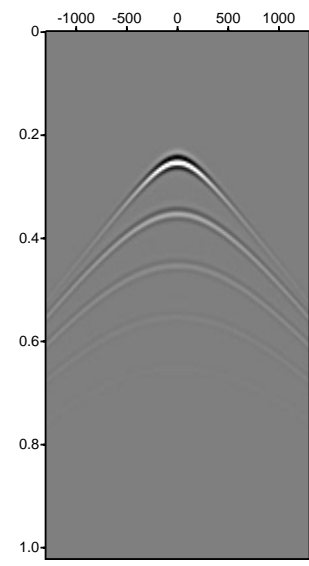

$\mathbf{T}$

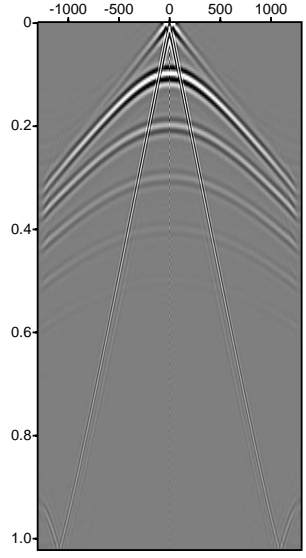

$\mathbf{R}^{H} \mathbf{R}$

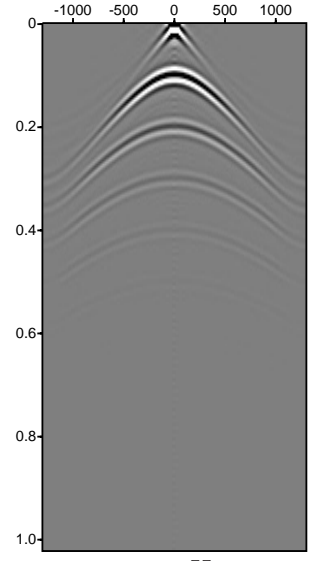

$\mathbf{T}^{H} \mathbf{T}$

Figure 2: Reflection and transmission data and correlation of transmission and reflection data in $x_{1}-t$ (i.e., one column of the data matrix, transformed back to time). The internal multiple train is clearly visible on the correlation of the transmission and reflection data.

which corresponds to a certain eigenvalue number $l$, to ray parameter $p$ with $\frac{1}{\omega}\left(k_{x}=p \omega\right)$. The scaling of the eigenvalues for $3 \mathrm{D}$ media remains to be investigated.

From equation (10) $\mathcal{R}\left\{\mathcal{A}_{i}\right\}$ can be calculated using: $2 \mathcal{R}\left\{\mathcal{A}_{i}\right\}=-\ln \left(\Lambda_{c, i}^{H} \Lambda_{c, i}\right)$. Reconstruction of $\mathcal{A}_{i}$ from its real part is possible because $\mathcal{A}_{i}$ is a causal function and with the definition of the analytic signal it can be reconstructed (see Bracewell (1986) for more details). To summarize the procedure, the following steps must be taken to compute the transmission coda from reflection data:

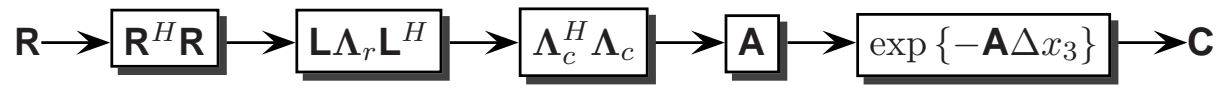

where $\boldsymbol{\Lambda}_{r}$ contains the eigenvalues of $\mathbf{R}^{H} \mathbf{R}$ and $\mathbf{I}-\boldsymbol{\Lambda}_{r}=\boldsymbol{\Lambda}_{c}^{H} \boldsymbol{\Lambda}_{c}$.

The inverse of the transmission coda (see also Herman (1992), Wapenaar and Hermann (1993)), in combination with an inverse primary propagator, may be used in seismic reflection imaging to obtain an image in which the internal multiple scattering effects are suppressed. In this approach the inverse primary propagator is estimated from the traveltime data (as usual), whereas the inverse transmission coda is obtained from the cross-correlation of the reflection measurements.

For comparison, in the imaging scheme proposed by Weglein et al. (2000), the full inverse operator (primaries as well as internal multiples) is estimated directly from the reflection measurements. The advantages and disadvantages of both methods with respect to accuracy, stability, etc. remain to be investigated.

\section{Results for point source in 1D medium}

For point sources in 1D media $\mathbf{R}^{H} \mathbf{R}$ is Hermitian and Toeplitz. Using the Toeplitz matrix as a basis to construct a circulant matrix, an approximation of its eigenvalues can be obtained by using the Fourier transform.

A $1 D 3$ layer medium is chosen with layer velocities 1000,4000 and $1000 \mathrm{~m} / \mathrm{s}$ and interface depths $0,200,400$ $\mathrm{m}$. The middle layer, thickness of $200 \mathrm{~m}$, will give internal multiples which are $0.1 \mathrm{~s}$. apart on the zero-offset trace. The reflection and transmission data, together with their correlation, are shown in figure(2).

The eigenvalues of $\mathbf{T}^{H} \mathbf{T}=\mathbf{I}-\mathbf{R}^{H} \mathbf{R}$ as function of the ray parameter $p$ are shown in figure (3a,b and c). The eigenvalues were computed in different ways. The Hermitian Toeplitz matrix can be transformed into a circulant matrix and the Fourier transform can then be used to calculate the eigenvalues (result shown in figure (3a)). The Lapack routine zheevx is also used to calculate the eigenvalues of the circulant matrix and give, as expected, the same results (figure (3b)) as obtained with the Fourier transform. However, after the eigenvalue decomposition the eigenvalues must be mapped to corresponding wavenumbers to be able to transform the results to the ray-parameter domain. The mapping from eigenvalues to wavenumber requires a sorting of the eigenvalues. The sorting used to obtain figure (3b) is based on the frequency content of the 


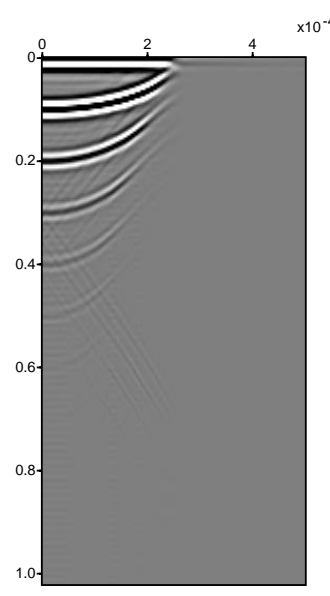

a) FFT of circulant

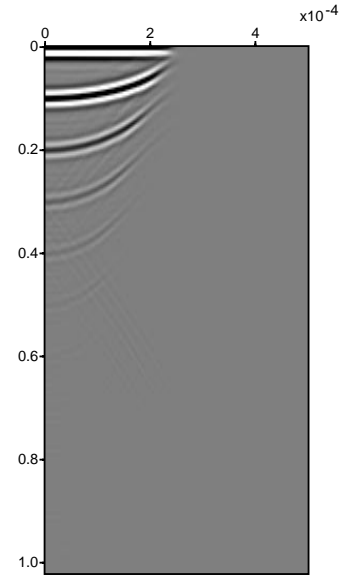

b) Lapack of circulant

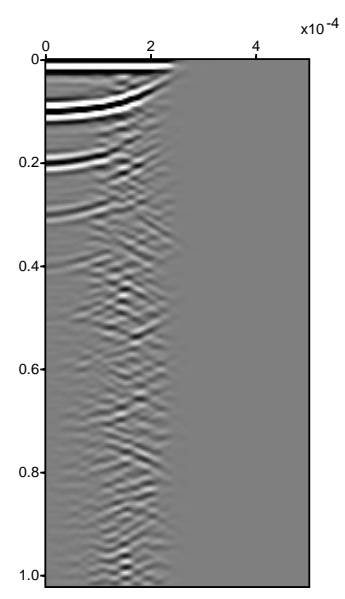

c) Lapack of Hermitian

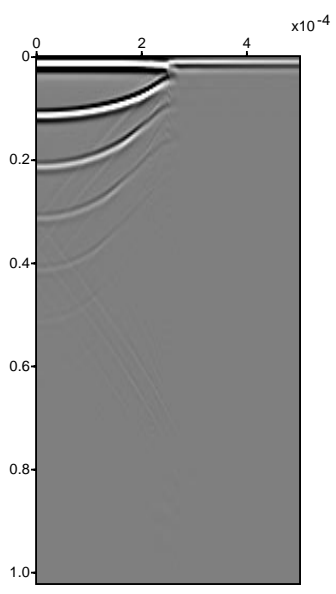

d) Coda from circulant

Figure 3: Eigenvalues $\left(\boldsymbol{\Lambda}_{t}\right)$ of correlation of transmission response in $\tau-p$ domain using FFT's (a) and Lapack routines (b) for a circulant matrix based on the Toeplitz matrix $\mathbf{T}^{H} \mathbf{T}$. The eigenvalue decomposition of the Hermitian matrix $(c)$ is done with Lapack. The coda operator $(d)$ is calculated from the eigenvalue decomposition based on the circulant matrix.

eigenvectors.

The third way of computing eigenvalues of Hermitian matrix are computed with the same Lapack routine zheevx and the results are shown in figure (3c). Note the effects of the edges of the matrix on the eigenvalue result.

Figure (3d) shows the computed coda operator from the eigenvalues of the circulant matrix. To compute the coda the logarithm of the eigenvalues, the diagonal of $\boldsymbol{\Lambda}_{c}^{H} \boldsymbol{\Lambda}_{c}$, is taken yielding $-2 \mathcal{R}\left\{\mathcal{A}_{i}\right\}$. From the real part of $\mathcal{A}_{i}$ the causal function is calculated using the Hilbert transform and the result is inserted into equations (6) and (7) to calculate the coda.

\section{Conclusions}

The implicit relation between correlated reflection and correlated transmission data can be used to estimate the transmission coda for 3D inhomogeneous media. The used assumptions on the transmission operator led to an eigenvalue problem. We demonstrated the solution of this general formulation for 1D media. Further investigation is needed in order to understand the eigenvalue decomposition in laterally varying media and the influence of the acquisition geometry.

\section{References}

Berkhout, A. J. (1982). Imaging of acoustic energy by wave field extrapolation (2nd edition). Elsevier, Amsterdam.

Bracewell, R. N. (1986). The Fourier transform and its applications, second edition. McGraw-Hill, Singapore.

Herman, G. C. (1992). Estimation of the inverse acoustic transmission operator of a heterogeneous region directly from its reflection operator. Inverse Problems, 8:559-574.

O’Doherty, R. and Anstey, N. (1971). Reflections on amplitudes. Geophysical Prospecting, 19:430-458.

Wapenaar, C. P. A. and Grimbergen, J. L. T. (1996). Reciprocity theorems for one-way wave fields. Geophysical Journal International, 127:169-177.

Wapenaar, C. P. A. and Hermann, F. J. (1993). True amplitude migration taking fine-layering into account, pages 653-656. Soc. of Expl. Geophys.

Weglein, A., Matson, K., Foster, D., Carvalho, P., Corrigan, D., and Shaw, S. (2000). Imaging and inversion at depth without a velocity model. In $70^{t} h$ Annual Internat. Mtg., Soc. Expl. Geophys., Expanded Abstracts, page Session: MIG 10.3. Soc. Expl. Geophys. 